\title{
Pseudotumor Cerebri as the First Manifestation of Juvenile Systemic Lupus Erythematosus
}

\author{
Seyed-Reza Raeeskarami, ${ }^{1}$ Leila Shahbaznejad, ${ }^{2,}{ }^{*}$ Raheleh Assari, ${ }^{2}$ and Yahya Aghighi ${ }^{2}$ \\ ${ }^{1}$ Department of Pediatrics, Pediatric Rheumatology Research Group, Rheumatology Research Center, Tehran University of Medical Sciences, Tehran, IR Iran \\ ${ }^{2}$ Department of Pediatrics, Tehran University of Medical Sciences, Tehran, IR Iran \\ "Corresponding author: Leila Shahbaznejad, Valiye-Asr Hospital, Imam Hospital Complex, Tehran, IR Iran. E-mail: leilashahbaznezhad@yahoo.com
}

Received 2015 December 27; Revised 2016 March 09; Accepted 2016 April 09.

\begin{abstract}
Introduction: Headache is a common neuropsychiatric manifestation of juvenile systemic lupus erythematous (JSLE). Pseudotumor cerebri (PTC) is an uncommon cause of headache, presenting especially in active JSLE. In this paper, we report a case of missed intractable headache that was eventually diagnosed as PTC and presented as the first manifestation of JSLE.

Case Presentation: A 9-year-old girl with a history of progressive headache for four months, fever, fatigue, myalgia, arthralgia, small-joint arthritis of the hands, and recent diplopia was referred to our clinic. The diagnosis of PTC in the background of JSLE was made based on her history, physical examination, positive laboratory findings, and increased intracranial pressure, with normal neuroimaging. Treatment with high-dose prednisolone led to dramatic resolution of the headache.

Conclusions: It seems that a complete neurologic examination in newly diagnosed SLE patients is mandatory, especially in the presence of any neuropsychiatric manifestations, such as headache.
\end{abstract}

Keywords: Lupus Erythematosus, Pseudotumor Cerebri, Headache

\section{Introduction}

Headache is a common neuropsychiatric manifestation in juvenile systemic lupus erythematosus (JSLE), occurring in approximately $38.3 \%$ of JSLE patients and $72.5 \%$ of neuropsychiatric SLE (NPSLE) patients (1). Pseudotumor cerebri (PTC) is an uncommon cause of headache, presenting especially in active JSLE (2). Moreover, there are rare published reports of PTC as the first manifestation of JSLE. In this paper, we report a case of missed intractable headache that was eventually diagnosed as PTC and that presented as the first manifestation of JSLE.

\section{Case Presentation}

A nine-year-old girl, the third child of nonconsanguineous parents, was referred to our clinic with severe headache and diplopia. The headache was localized in the right fronto-occipital region with spread to the neck and shoulders. Transient diplopia was her most recent complaint. According to the patient's previous records, symptoms of low-grade fever, fatigue, myalgia, arthralgia, mild headache, and small-joint arthritis of the hands had been present for four months. She had no photosensitivity, malar rash, skin lesions, oral ulcers, hair loss, or weight loss. Laboratory tests at that time showed mild cytopenia, with platelet count (Plt) of $249,000 / \mu \mathrm{L}$. Her one-hour erythrocyte sedimentation rate (ESR) was $20 \mathrm{~mm} / \mathrm{h}$ and C-reactive protein (CRP) was negative. There were elevated transaminases, with alanine transaminase (ALT) of $108 \mathrm{U} / \mathrm{mL}$ and aspartate transaminase (AST) of $70 \mathrm{U} / \mathrm{mL}$, and normal thyroid function tests, with T4 of 8 $\mu \mathrm{g} / \mathrm{dL}$, thyroid stimulating hormone (TSH) of $4 \mathrm{mIU} / \mathrm{L}$. The results also showed negative antiphospholipid antibodies and venereal disease research laboratory (VDRL) testing, positive anti-nuclear antibody (ANA), negative anti-double stranded DNA (anti-dsDNA) of $69 \mathrm{U} / \mathrm{mL}$ (positive > 100), decreased complement levels (C3, C4), and normal urinalysis. The results were compatible with juvenile-onset SLE, but optimal follow-up and treatment had not been performed.

After two months, her headache gradually progressed, but showed a good response to aspirin $325 \mathrm{mg} 3$ - 4 times per day, given by her parents. The neurologic findings included bilateral papilledema, bilateral 6th cranial nerve palsy, normal magnetic resonance imaging (MRI) and magnetic resonance angiogram (MRA), and high cerebrospinal fluid (CSF) opening pressure ( $55 \mathrm{~cm} \mathrm{H}_{2} \mathrm{O}$ at the first visit, 32 $\mathrm{cm} \mathrm{H}_{2} \mathrm{O}$ at the 7th day, and normal at the 10th day). CSF analysis was within normal limits, with sugar of $46 \mathrm{mg} / \mathrm{dL}$, pro- 
tein of $33 \mathrm{mg} / \mathrm{dL}$, lactate dehydrogenase (LDH) of $64 \mathrm{U} / \mathrm{mL}$, and zero WBCs or red blood cells (RBCs). Acetazolamide was prescribed for PTC, but no significant clinical response was seen. Her parents and other siblings had no history of disease; however, her 50-year-old aunt (mother's sister) suffered from lupus nephritis and her five-year-old cousin (daughter of mother's brother) had been treated for myasthenia gravis three years earlier. In the patient's past medical history, levothyroxine had been prescribed for hypothyroidism since the age of four, with good control.

The patient was admitted to our hospital with symptoms of severe headache, diplopia, fatigue, malaise, and new-onset hair loss. Physical examination showed that she was alert, with an axillary temperature of $38.3^{\circ} \mathrm{C}$, weight of $30 \mathrm{~kg}$, height of $137 \mathrm{~cm}$, blood pressure of 95/60 $\mathrm{mmHg}$, and heart rate of 100 beats $/ \mathrm{min}$. She had bilateral papilledema, bilateral 6th nerve palsy, mild organomegaly, and smalljoint arthritis of the hands.

The laboratory data at the time of admission showed mild leukopenia and lymphopenia, without thrombocytopenia (WBC 4300/ $\mu \mathrm{L}, 42 \%$ lymphocytes, Hgb $12.7 \mathrm{~g} / \mathrm{dL}$, and Plt $307,000 / \mu \mathrm{L})$. She had positive ANA $(103 \mathrm{U} / \mathrm{mL}$, positive $>42$ ) and increased anti-dsDNA ( $497 \mathrm{U} / \mathrm{mL}$, positive $>$ 100). Biochemical markers of renal function (blood urea nitrogen and creatinine) and the urinalysis were within normal limits, without any proteins or RBCs. The mild hypertransaminasemia detected in the previous month decreased to near-normal values. Complement (C3, C4) levels continuously showed low titers. Autoantibodies to ribosomal P proteins (anti-Rib-P) had borderline values according to the laboratory references. Although a high titer of antithyroid peroxidase antibody (anti-TPOAb) was detected (270 $\mathrm{IU} / \mathrm{mL}$, positive $>40$ ), thyroid function tests showed good control.

According to the patient's history, physical examination findings of arthritis, and positive laboratory findings (positive ANA test, increased anti-dsDNA Ab, and decreased complement [C3, C4] levels), the patient fulfilled four SLE criteria (three laboratory criteria and one clinical criterion) (3). She was therefore treated for SLE with acetazolamide, prednisolone ( $1.5 \mathrm{mg} / \mathrm{kg} /$ day $)$, and hydroxychloroquine. After 24 hours, the headache dramatically subsided and the patient's condition improved. She was discharged in good general condition. Acetazolamide was prescribed with a tapering dose for two weeks. After six months, the patient was in good condition, without any complaints of headache or diplopia.

\section{Discussion}

Headache is one of the most common complaints in SLE patients. The causes of headache in SLE patients are rarely detectable, but PTC as an infrequent cause should be considered (4). PTC was characterized by increased intracranial pressure (ICP) of $>20 \mathrm{~cm} \mathrm{H}_{2} \mathrm{O}$, with normal neurologic findings except for 6th nerve palsy and papilledema. The CSF analysis and brain imaging were normal (5). The most serious complication of PTC is blindness due to severe papilledema (5).

There are sporadic reports of concomitant SLE and PTC in adults, and more rarely in children (6-10). Moreover, in a few cases, PTC was reported as a first manifestation of SLE in children (6-10). Most of these patients had prolonged headache, with the interval between the onset of headache and the diagnosis of SLE being approximately two months. The headache usually subsides immediately after treatment with high-dose corticosteroids. On the other hand, delayed diagnosis or treatment (such as in our patient) could increase the ICP, fulfilling the PTC criteria (5).

In general, PTC is not a common manifestation in SLE patients. It has been reported in approximately $17 \%$ of SLE patients with intractable headaches (2). However, nausea, vomiting, and blurred vision are common in these cases (2, 6-10).

In a report of SLE patients, Kim and coworkers (2) focused on the characteristics of PTC during the course of SLE. They noted that most PTC manifestations occur during the active phase of SLE. The pathogenesis of PTC in SLE patients remains unknown. In some patients, venous thrombosis occurs in a hypercoagulable state of antiphospholipid antibody syndrome. In approximately 58\% of SLE patients with PTC, thromboembolic events and high titers of anti-cardiolipin antibody have been reported (11). In our patient, these antibodies were negative, and MRI and MRV were normal, so a thrombotic event could not be the cause of PTC in this patient. On the other hand, in the active phase of SLE, immune complex, direct antibodies, or vasculitis may affect the function of arachnoid villi, disturbing the absorption of CSF and causing PTC (12). This mechanism may explain the occurrence of PTC in our case.

Fragoso-Loyo et al. reported high levels of some cytokines, chemokines, and inflammatory molecules in the CSF of SLE patients with intracranial hypertension and intractable nonspecific headache; however, after approximately six months, when the headaches were resolved, these returned to normal limits (13).

In addition, anti-Rib-P antibodies and lupus nephritis may accompany PTC (2). Our case had no renal complications, but low titers of serum anti-Rib-P antibodies were detected. There are some discrepancies in the literature about the presence of autoantibodies in such cases. Pradhan et al. reported that anti-Rib-P was found in $28.2 \%$ of SLE patients with neuropsychiatric involvement (NPSLE), 
whereas $20 \%$ of SLE patients without neuropsychiatric involvement also had the same antibody. Therefore, anti-Rib$P$ is not a specific test for the diagnosis of NPSLE (14). Our patient had no significant amount of anti-Rib-P antibody at the time of presentation. However, after a reasonable time, any changes in auto-antibody titers should be followed.

Acetazolamide resulted in no clinical improvement in the patient's condition. Corticosteroids are the main treatment for PTC in SLE patients, either as intravenous methyl prednisolone pulse therapy or as high-dose oral prednisolone (2, 6-10). Treatment with prednisolone dramatically improved our patient's PTC after approximately 24 hours.

In conclusion, it seems that a complete neurologic examination in newly diagnosed SLE patients is mandatory, especially in the presence of any neuropsychiatric manifestations, such as headache.

\section{References}

1. Torreggiani S, D’Errico M, Di Landro G, Cuoco F, Petaccia A, Cappellari A, et al. Neuropsychiatric manifestations in juvenile systemic lupus erythematosus: what's the weight of headache?. Ped Rheum. 2014;12(Suppl 1):P310. doi: 10.1186/1546-0096-12-s1-p310.

2. Kim JM, Kwok SK, Ju JH, Kim HY, Park SH. Idiopathic intracranial hypertension as a significant cause of intractable headache in patients with systemic lupus erythematosus: a 15-year experience. Lupus. 2012;21(5):542-7. doi: 10.1177/0961203311435267. [PubMed: 22300830].

3. Petri M, Orbai AM, Alarcon GS, Gordon C, Merrill JT, Fortin PR, et al. Derivation and validation of the Systemic Lupus International Collaborating Clinics classification criteria for systemic lupus erythematosus. Arthritis Rheum. 2012;64(8):2677-86. doi: 10.1002/art.34473. [PubMed: 22553077].

4. Hanly JG, Urowitz MB, O'Keeffe AG, Gordon C, Bae SC, SanchezGuerrero J, et al. Headache in systemic lupus erythematosus: re- sults from a prospective, international inception cohort study. Arthritis Rheum. 2013;65(11):2887-97. doi: 10.1002/art.38106. [PubMed: 24166793].

5. Hacifazlioglu Eldes N, Yilmaz Y. Pseudotumour cerebri in children: etiological, clinical features and treatment modalities. Eur J Paediatr Neurol. 2012;16(4):349-55. doi: 10.1016/j.ejpn.2011.09.002. [PubMed: 22050895].

6. Liyanage G, Herath HMD. Pseudo tumour cerebri in systemic lupus erythematosus. Sri Lanka J Child Health. 2008;37(2) doi: 10.4038/sljch.v37i2.99.

7. Sbeiti S, Kayed DM, Majuri H. Pseudotumour cerebri presentation of systemic lupus erythematosus: more than an association. Rheumatology (Oxford). 2003;42(6):808-10. doi: 10.1093/rheumatology/keg200. [PubMed: 12771445].

8. Farhadi A, Haghshenas M. Pseudo tumor cerebri as the major manifestation of systemic lupus er y thematosus in a case of fever of unde termined origin. MJIRI. 1997;11(3):259-61.

9. Kuyucu S, Argin A, Kuyucu N, Ozen S. Systemic lupus erythematosus presenting with pseudotumor cerebri: a rare association. Turk J Pediatr. 2007;49(1):98-101. [PubMed: 17479654].

10. Maloney K. Idiopathic intracranial hypertension as an initial presentation of systemic lupus erythematosus. BMJ Case Rep. 2013;2013 doi: 10.1136/bcr-2013-010223. [PubMed: 23943808].

11. Parnass SM, Goodwin JA, Patel DV, Levinson DJ, Reinhard JD. Dural sinus thrombosis: a mechanism for pseudotumor cerebri in systemic lupus erythematosus. J Rheumatol. 1987;14(1):152-5. [PubMed: 3553588].

12. Horoshovski D, Amital H, Katz M, Shoenfeld Y. Pseudotumour cerebri in SLE. Clin Rheumatol. 1995;14(6):708-10. [PubMed: 8608694].

13. Fragoso-Loyo H, Atisha-Fregoso Y, Llorente L, Sanchez-Guerrero J. Inflammatory profile in cerebrospinal fluid of patients with headache as a manifestation of neuropsychiatric systemic lupus erythematosus. Rheumatology (Oxford). 2013;52(12):2218-22. doi: 10.1093/rheumatology/ket294. [PubMed: 24026247].

14. Pradhan V, Patwardhan M, Rajadhyaksha A, Dhawale N, Ghosh K. Neuropsychiatric manifestations and associated autoantibodies in systemic lupus erythematosus patients from Western India. Rheumatol Int. 2015;35(3):541-5. doi: 10.1007/s00296-014-3114-z. [PubMed: 25119833]. 